\title{
IgG4 Related Disease as a Differential Diagnosis in Multi-Organ Systemic Disease
}

\author{
Mohammad Al Mousa, Fahad Al Muhanna, Ahmad Al Shami, Yaser M. Ali \\ Department of Medicine, Mubarak Al Kabeer Hospital, Kuwait City, Kuwait \\ Email: almousa1989@gmail.com
}

How to cite this paper: Al Mousa, M., Al Muhanna, F., Al Shami, A. and Ali, Y.M. (2021) IgG4 Related Disease as a Differential Diagnosis in Multi-Organ Systemic Disease. Open Journal of Rheumatology and Autoimmune Diseases, 11, 19-27. https://doi.org/10.4236/ojra.2021.111003

Received: November 9, 2020

Accepted: February 5, 2021

Published: February 8, 2021

Copyright $\odot 2021$ by author(s) and Scientific Research Publishing Inc. This work is licensed under the Creative Commons Attribution International License (CC BY 4.0).

http://creativecommons.org/licenses/by/4.0/

\begin{abstract}
IgG4 related disease is a relatively recently recognized systemic entity, that has been observed in nearly every organ system, with similar histopathological features across all identified organs. IgG4 is an antibody molecule accounting for less than $5 \%$ of the total IgG count in a healthy person. The molecule is implicated in numerous autoimmune diseases; however its role in IgG4 related disease, whether primary or secondary, remains unknown. The gold standard in the diagnosis of this condition is demonstration of organ involvement from biopsy specimens. In this case report, the patient had exhibited symptoms related mainly to the gastrointestinal tract for 12 years prior to his diagnosis of IgG4 related disease. After this patient's diagnosis of IgG4 related disease, he was commenced on Rituximab, a monoclonal antibody, which was successful in subsiding his symptoms and he has remained symptom free for the past two years of follow up since commencing treatment and hadn't developed any complications of the disease nor any complications related to treatment. IgG4 related disease is a systemic autoimmune disease that involves multiple organs $60 \%-90 \%$ of the time, therefore clinicians should be aware that IgG4 related disease can mimic some autoimmune rheumatic diseases and place it as a differential diagnosis when multi-organ disease with systemic features is involved.
\end{abstract}

\section{Keywords}

IgG4 Related Disease, Autoimmune Disease, Rituximab

\section{Introduction}

IgG4 related disease (IgG4-RD) is a spectrum of newly described conditions that exhibit the features of: tumefactive lesions, a dense lymphoplasmacytic infiltrate rich in IgG4 positive plasma cells, storiform fibrosis, and more often than not, elevated serum IgG4 antibodies [1]. It was only at the time when extra-pancreatic 
manifestations were found in patients with autoimmune pancreatitis in 2003, that the disease was recognized as a systemic condition. The disease has been studied and observed in nearly every organ system, with similar histopathological features across all identified organs, regardless of the site of disease [1] [2] [3] [4] [5]. Organ systems like the biliary tree, salivary glands, periorbital tissues, kidneys, lungs, lymph nodes, meninges, aorta, prostate, thyroid gland, pericardium, and skin have all been identified in IgG4-related disease.

Many well-known medical conditions that have been described in the literature, are now believed to be part of the spectrum of IgG4-related disease. These conditions include Mikulicz's syndrome, Kuttner's tumor, and Riedel's thyroiditis, to name a few.

IgG4 is an antibody molecule accounting for less than $5 \%$ of the total IgG count in a healthy person, making it the least abundant antibody in the IgG subclass [6] [7]. Even with this molecule's implication in many autoimmune conditions, the behavior of IgG4 in vivo as well as its role in IgG4 related disease whether being primary or secondary, remains unknown.

The cornerstone method for diagnosis of IgG4 disease is the histopathological analysis of sampled biopsy specimens. Elevated concentrations of IgG4 in tissue and serum are helpful but are not specific diagnostic markers for IgG4 related disease [8] [9]. Therefore, correlation with specific histopathological findings is essential if plausible, regardless of the serum IgG4 concentration, the number of IgG4 positive plasma cells in the tissue, or the ratio of IgG4/IgG in tissue [1]. However, obtaining the specific histopathological findings is not always feasible. This is mainly due to the variety of ways and multiple organs this disease process can present with, rendering suspicion of the disease less likely in its initial phases. As the disease progresses, when there is a late phase of organ involvement, there will be fewer plasma cells for analysis of the IgG4 molecule. In our case, the patient had exhibited symptoms related mainly to the gastrointestinal tract for 12 years prior to a formal diagnosis. With the nature of his history of illness, which involved multiple organs, that were not identifiable to a single unifying systemic disorder, accompanied with the extremely high IgG4 levels, a diagnosis of IgG4 related disease had been made.

A study was conducted by Carruthers MN, Khosroshahi A, Augustin T, et al., over a 10-year period (2001-2011) on 380 patients, 72 of whom had either probable or definitive IgG4-RD, 65 had elevated serum IgG4 concentrations, giving a sensitivity of $90 \%$. Among the 308 subjects without IgG4-RD, 125 had elevated IgG4 and 183 had normal IgG4 concentrations, for a specificity of $60 \%$ [10].

\section{Case Report}

Mr. A is a 62-year-old from Kuwait. The patient's history starts from the time he was 50 years of age. At the time leading up to the diagnosis of IgG4 related disease, he went to his healthcare provider with a three-month history of decreased appetite, night sweats, change in bowel associated with weight loss. The weight 
loss was quantified as ten kilograms without any intention of weight loss. The patient never complained of bleeding per rectum, melena or any abdominal pain. Historically the patient had a recurrent urticarial rash in the upper limbs and trunk, which appeared and could last for weeks, before self-resolving. Furthermore, he also had recurrent angioedema which resolved on antihistamine medications. He had no symptoms of hemoptysis, epistaxis, nasal polyp nor bronchial asthma, or any allergies except for the fact that he noticed that multivitamins preparations have exacerbated the rash. He reports no history of joint stiffness, no pain, no bleeding tendency, and no hematuria. The patient is married and lives with his extended family and works an office job in the ministry of education as a director of a department. He was an ex-smoker with a twelve-year pack smoking history. He travels annually to Germany during his vacation period.

On examination, the patient showed evidence of cervical, supraclavicular and axillary palpable lymph nodes, with sizes varying from $2-4 \mathrm{~cm}$. He also showed evidence of hepatosplenomegaly upon abdomen examination, without tenderness, any scratch marks or any palpable inguinal lymph nodes. Cardiovascular, respiratory and neurological examination revealed no abnormalities. The patient's sociodemographic and clinical features are summarized in Table 1.

Basic laboratory blood tests were obtained as part of the initial investigations, including a full blood count, shown in Table 2 below. His electrocardiogram showed a normal sinus rhythm, and his routine initial chest radiograph, shown in Figure 1 below, was unremarkable.

Table 1. Patient's sociodemographic and clinical features summarized.

Patient's Sociodemographic Characteristics.

- 62-year-old male

- Married

- Works in an office in the local ministry of education.

- Former cigarette smoker, with a 12-year pack history.

- Non-alcohol consumer.

- No IVDU.

Table 2. Complete blood count result (from patient's own medical records).

\begin{tabular}{ccc}
\hline White Blood Cell (WBC) & $8 \times 10^{9} / \mathrm{L}$ & $3.7-10$ \\
Hemoglobin & $105 \mathrm{~g} / \mathrm{L}$ & $130-170$ \\
Mean corpuscular volume (MCV) & $68 \mathrm{fL}$ & $83-101$ \\
Mean corpuscular hemoglobin (MCH) & $21 \mathrm{pg}$ & $27-32$ \\
Platelets & $390 \times 10^{9} / \mathrm{L}$ & $130-430$ \\
Neutrophil & $4.4 \times 10^{9} / \mathrm{L}$ & $1-7.6$ \\
Lymphocytes & $3.3 \times 10^{9} / \mathrm{L}$ & $1-3$ \\
Eosinophils & $0.11 \times 10^{9} / \mathrm{L}$ & $0.02-0.5$ \\
\hline
\end{tabular}


A blood film showed hypochromic microcytic anemia, anisopoikilocytosis including a presence of target cells and moderate rouleaux formation.

The erythrocyte sedimentation rate was $120 \mathrm{~mm} / \mathrm{hr}(0-20)$, total protein 107 g/L (66 - 83), abumin 21 g/L (35 - 52), urea, creatinine, liver enzymes, electrolytes, calcium, lactate, and coagulation profile were all within normal limits.

A virology screen was done and was negative for Cytomegalovirus, Epstein-Barr virus, the human immunodeficiency virus, as well as for hepatitis B and C. A purified protein derivative (PPD) skin test was $15 \mathrm{~mm}$, with a negative Polymerase chain reaction (PCR) for tuberculosis, and the Gamma Quantiferon test also came back as negative.

The Immunology Screen was negative for Anti CCP, anti dsDNA, Anti proteinase-3 antibody, anti-neutrophil cytoplasmic antibody, anti myeloperoxidase antibody. However, it was positive for Antinuclear Antibody (ANA), in a homogenous pattern in a 1:640 titer. Serum Immunoglobulin levels were also obtained, shown in Table 3 below, which had shown a very high IgG4 level.

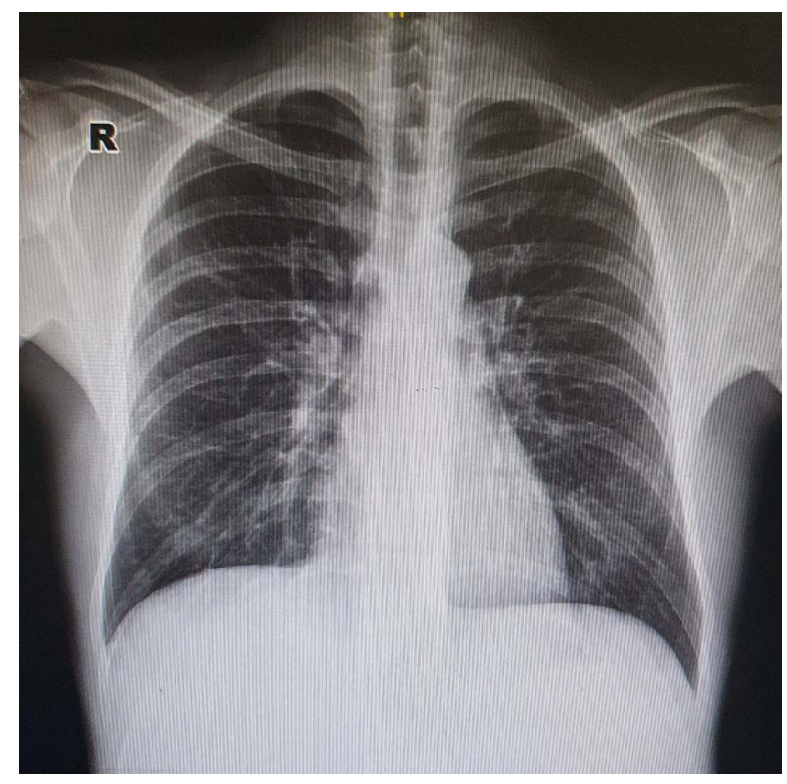

Figure 1. Chest X-ray obtained on first visit with no abnormalities (from patient's own medical records).

Table 3. Serum Immunoglobulin levels (obtained from patient's own medical records).

\begin{tabular}{ccc}
\hline Immunoglobulin IgG & $24 \mathrm{~g} / \mathrm{L}$ & Reference range: \\
& & $6-16$ \\
IgG1 & $6929 \mathrm{mg} / \mathrm{L}$ & Reference range: \\
& & $3824-9286$ \\
IgG2 & Reference range: \\
& $7860 \mathrm{mg} / \mathrm{L}$ & $2418-7003$ \\
IgG3 & $1200 \mathrm{mg} / \mathrm{L}$ & Reference range: \\
& & $218.2-1761$ \\
IgG4 & Reference range: \\
& $6130 \mathrm{mg} / \mathrm{L}$ & $39-864$ \\
\hline
\end{tabular}


Imaging was requested in the form of Computed Tomography of the chest, abdomen, and pelvis, which showed bilateral cervical, supraclavicular, axillary lymphadenopathy, diffuse abdominal lymphadenopathy, hepatosplenomegaly. Figure 2 below shows CT image slices of the abdomen and pelvis. Endoscopy showed evidence of Barrett's esophagus, gastritis and duodenitis. A colonoscopy revealed a rectal polyp, terminal ileitis, ileocecal ulcer, and two rectal ulcers size of $3 \mathrm{~cm}$ each. Multiple biopsies were taken from the lesser curvature, antrum, esophagus, rectum, terminal ileum and all were reported as inconclusive in relation to any systemic disorder. A right cervical lymph node was biopsied, resulting in a reactive lymph node.

With the clinical features as well as laboratory and imaging findings pointing towards a multiorgan systemic disease, this patient was ultimately diagnosed with IgG4-related disease. The treatment used to control his disease was Rituximab, which is a chimeric monoclonal antibody against the protein CD20. Following his diagnosis and commencement of treatment, the patient has been on the same treatment for the past two years. With follow up, since starting treatment, he has been in remission and has remained symptom free. Furthermore, upon follow up he has not developed any further complications related to his disease process, nor has he developed complications related to his treatment.

\section{Discussion}

\section{Overview of manifestations:}

IgG4-related disease (IgG4-RD) can involve one or multiple organs, and manifestations of this disease have been demonstrated in nearly every organ system [1] [11] [12] [13].

Multiple organs are affected in 60 to 90 percent of patients with IgG4-RD [14] [15].
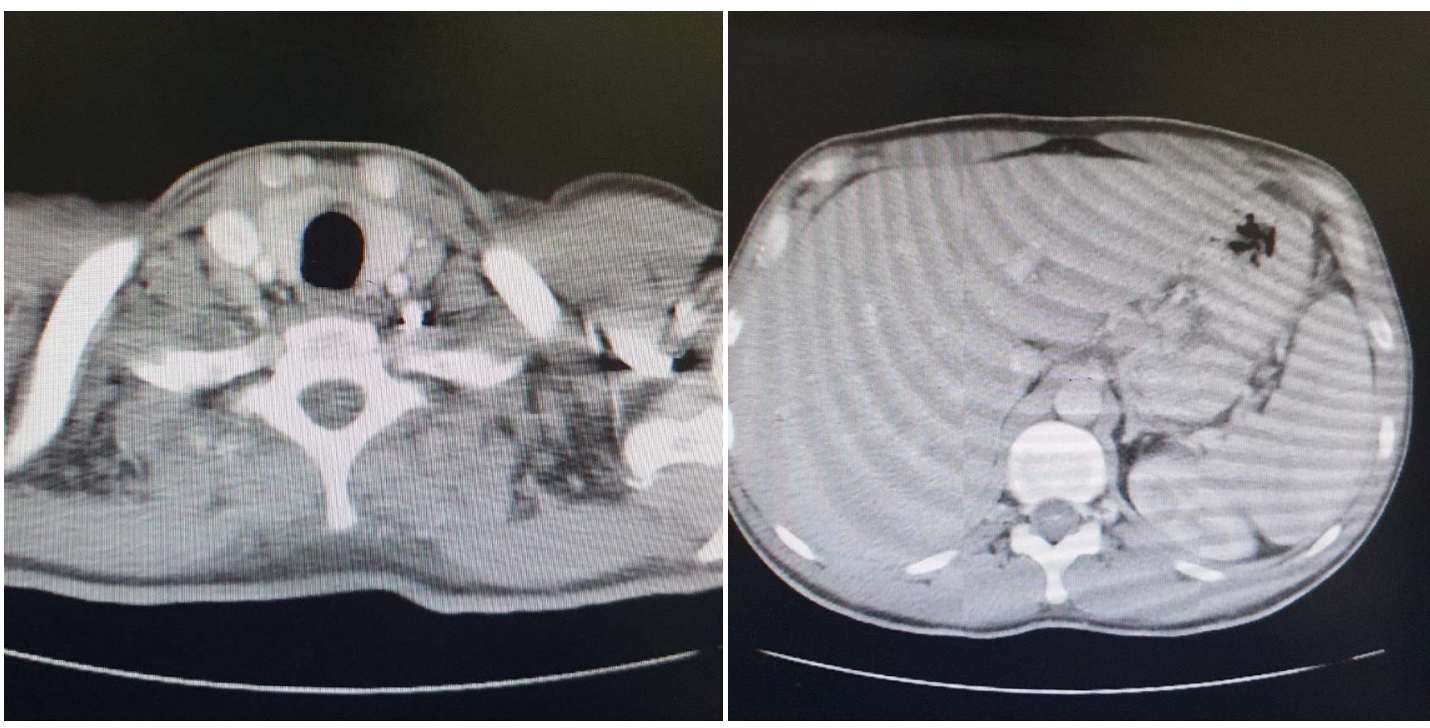

Figure 2. Some image slices of the computed tomography images of the abdomen and pelvis (obtained from patient's own medical records). 
Despite the multiorgan nature of the condition, four main clinical phenotypes (i.e., patterns of organ involvement) have been identified using latent class analysis, a form of cluster analysis, to categorize 765 patients from two large cross-sectional cohorts, which included patients with IgG4-RD from many different centers [16]. Each group, which comprised approximately 20 to 30 percent of all the patients in the cohorts, was identified and named based upon the most common manifestations and involved organs in the group.

These four phenotypes included the following groups:

Group 1-Pancreato-hepato-biliary disease

Group 2-Retroperitoneal fibrosis and/or aortitis

Group 3-Head- and neck-limited disease

Group 4-Classic Mikulicz syndrome with systemic involvement

The affected tissues share particular pathologic features, although some of them, such as storiform fibrosis, are not encountered frequently in certain tissues, including salivary glands and lymph nodes [17].

Lymphadenopathy is common complaint and physical finding, and symptoms of asthma or allergy are present in approximately 40 percent of patients diagnosed with IgG4-RD. Patients often feel well at the time of diagnosis and generally lack fever [18].

Asymptomatic IgG4-related lymphadenopathy is common, occurring in 80 percent of patients with autoimmune pancreatitis (AIP) [19]. Lymphadenopathy is usually observed together with other clinical or laboratory manifestations of the syndrome, but may be the initial or only manifestation [19].

Biopsies of lymph nodes are often problematic to interpret with regard to the diagnosis of IgG4-RD because they seldom undergo the storiform fibrosis that is so highly characteristic of IgG4-RD, and large numbers of IgG4+ plasma cells can be found in multiple diseases in which IgG4-RD is not the diagnosis, resulting in poor specificity of this finding.

In a study of 114 patients with varied organ involvement, lymphadenopathy was present in 41 percent of patients [20]. Symptoms occasionally occur due to mass effect of the enlarging nodes; individual nodes are typically no more than 2 centimeters in diameter but may range up to 5 centimeters [20].

Multiple groups of lymph nodes are usually involved; the mediastinal, hilar, intraabdominal, and axillary are most common and can be readily seen upon scanning with gallium-67 [20].

The lymphadenopathy is generally non-tender and the nodes themselves are rubbery rather than hard.

Patients with lymphadenopathy may exhibit elevated serum IgG4, serum IgG and IgE, polyclonal hypergammaglobulinemia, and elevations in the erythrocyte sedimentation rate (ESR).

Patients with multiorgan disease often lose substantial amounts of weight-20 to 30 pounds (approximately 9 to $14 \mathrm{~kg}$ ) over months-before the correct diagnosis is identified. 
Clinicians should be alert to the possibility that IgG4-RD can mimic some autoimmune rheumatic diseases such as Sjögren's syndrome (SS), systemic lupus erythematosus (SLE), and granulomatosis with polyangiitis, as well as a number of other conditions

Additionally, IgG4-RD can overlap with other autoimmune diseases, including antiphospholipid syndrome and rheumatoid arthritis [11].

In skin disease, including a subset of cutaneous pseudolymphoma, the lesions typically appear on the scalp, face, neck, and pinna of the ear [21] [22] [23]. Papules, plaques, and nodules are common cutaneous manifestations, while macules and bullae are rarely seen [12].

IgG4-hepatopathy can also occur, resembling autoimmune hepatitis, and hepatic inflammatory pseudotumor [23] [24].

\section{Conclusion}

IgG4 related disease is a systemic autoimmune disease that involves multiple organs $60 \%-90 \%$ of the time. Many organ systems have been implicated to be affected by this disease process such as the biliary tree, salivary glands, periorbital tissues, kidneys, lungs, lymph nodes, meninges, aorta, prostate, thyroid gland, pericardium, and skin. It is also possible that IgG4 related disease can mimic and overlap with a number of important autoimmune and systemic diseases. Four main phenotypes of the disease have been identified, including: group 1: pancreato-hepato-biliary disease, group 2: retroperitoneal fibrosis and/or aortitis, group 3: head- and neck-limited disease, group 4: classic Mikulicz syndrome with systemic involvement. The mainstay in the diagnosis of this condition is via demonstration of histopathologic characteristics in biopsy specimens, as well as high levels of serum or tissue IgG4 levels to help guide towards the diagnosis in the appropriate clinical context.

\section{Acknowledgements}

The authors wish to thank Dr. Yaser Mustafa, consultant of rheumatology in Mubarak Hospital, and all the members of the department of rheumatology.

\section{Conflicts of Interest}

The authors declare no conflicts of interest regarding the publication of this paper.

\section{References}

[1] Kamisawa, T., Funata, N., Hayashi, Y., et al. (2003) A New Clinicopathological Entity of IgG4-Related Autoimmune Disease. Journal of Gastroenterology, 38, 982-984. https://doi.org/10.1007/s00535-003-1175-y

[2] Stone, J.H., Khosroshahi, A., Hilgenberg, A., Spooner, A., Isselbacher, E.M. and Stone, J.R. (2009) IgG4-Related Systemic Disease and Lymphoplasmacytic Aortitis. Arthritis \& Rheumatology, 60, 3139-3145. https://doi.org/10.1002/art.24798

[3] Dahlgren, M., Khosroshahi, A., Nielsen, G.P., Deshpande, V. and Stone, J.H. (2010) 
Riedel's Thyroiditis and Multifocal Fibrosclerosis Are Part of the IgG4-Related Systemic Disease Spectrum. Arthritis Care \& Research (Hoboken), 62, 1312-1318. https://doi.org/10.1002/acr.20215

[4] Saeki, T., Saito, A., Hiura, T., et al. (2006) Lymphoplasmacytic Infiltration of Multiple Organs with Immunoreactivity for IgG4: IgG4-Related Systemic Disease. Internal Medicine, 45, 163-167. https://doi.org/10.2169/internalmedicine.45.1431

[5] Kamisawa, T., Takuma, K., Egawa, N., Tsuruta, K. and Sasaki, T. (2010) Autoimmune Pancreatitis and IgG4-Related Sclerosing Disease. Nature Reviews Gastroenterology \& Hepatology, 7, 401-409. https://doi.org/10.1038/nrgastro.2010.81

[6] Aalberse, R.C., Stapel, S.O., Schuurman, J. and Rispens, T. (2009) Immunoglobulin G4: An Odd Antibody. Clinical \& Experimental Allergy, 39, 469-477. https://doi.org/10.1111/j.1365-2222.2009.03207.x

[7] Nirula, A., Glaser, S.M., Kalled, S.L. and Taylor, F.R. (2011) What Is IgG4? A Review of the Biology of a Unique Immunoglobulin Subtype. Current Opinion in Rheumatology, 23, 119-124. https://doi.org/10.1097/BOR.0b013e3283412fd4

[8] Strehl, J.D., Hartmann, A. and Agaimy, A. (2011) Numerous IgG4-Positive Plasma Cells Are Ubiquitous in Diverse Localised Non-Specific Chronic Inflammatory Conditions and Need to Be Distinguished from IgG4-Related Systemic Disorders. Journal of Clinical Pathology, 64, 237-243. https://doi.org/10.1136/jcp.2010.085613

[9] Sah, R.P. and Chari, S.T. (2011) Serologic Issues in IgG4-Related Systemic Disease and Auto-Immune Pancreatitis. Current Opinion in Rheumatology, 23, 108-113. https://doi.org/10.1097/BOR.0b013e3283413469

[10] Carruthers, M.N., Khosroshahi, A., Augustin, T., Deshpande, V. and Stone, J.H. (2015) The Diagnostic Utility of Serum IgG4 Concentrations in IgG4-Related Disease. Annals of the Rheumatic Diseases, 74, 14-18.

[11] Miyagawa-Hayashino, A., Matsumura, Y., Kawakami, F., et al. (2009) High Ratio of IgG4-Positive Plasma Cell Infiltration in Cutaneous Plasmacytosis-Is This a Cutaneous Manifestation of IgG4-Related Disease? Human Pathology, 40, 1269. https://doi.org/10.1016/j.humpath.2009.01.013

[12] Zen, Y., Fujii, T., Sato, Y., et al. (2007) Pathological Classification of Hepatic Inflammatory Pseudotumor with Respect to IgG4-Related Disease. Modern Pathology, 20, 884. https://doi.org/10.1038/modpathol.3800836

[13] Brito-Zerón, P., Ramos-Casals, M., Bosch, X. and Stone, J.H. (2014) The Clinical Spectrum of IgG4-Related Disease. Autoimmunity Reviews, 13, 1203.

https://doi.org/10.1016/j.autrev.2014.08.013

[14] Okazaki, K., Uchida, K., Koyabu, M., et al. (2011) Recent Advances in the Concept and Diagnosis of Autoimmune Pancreatitis and IgG4-Related Disease. Journal of Gastroenterology, 46, 277. https://doi.org/10.1007/s00535-011-0386-X

[15] Sah, R.P., Chari, S.T., Pannala, R., et al. (2010) Differences in Clinical Profile and Relapse Rate of Type 1 versus Type 2 Autoimmune Pancreatitis. Gastroenterology, 139, 140. https://doi.org/10.1053/j.gastro.2010.03.054

[16] Wallace, Z.S., Zhang, Y., Perugino, C.A., et al. (2019) Clinical Phenotypes of IgG4-Related Disease: An Analysis of Two International Cross-Sectional Cohorts. Annals of the Rheumatic Diseases, 78, 406. https://doi.org/10.1136/annrheumdis-2018-214603

[17] Cheuk, W. and Chan, J.K. (2010) IgG4-Related Sclerosing Disease: A Critical Appraisal of an Evolving Clinicopathologic Entity. Advances in Anatomic Pathology, 17, 303. https://doi.org/10.1097/PAP.0b013e3181ee63ce 
[18] Hamano, H., Arakura, N., Muraki, T., et al. (2006) Prevalence and Distribution of Extrapancreatic Lesions Complicating Autoimmune Pancreatitis. Journal of Gastroenterology, 41, 1197. https://doi.org/10.1007/s00535-006-1908-9

[19] Saegusa, H., Momose, M., Kawa, S., et al. (2003) Hilar and Pancreatic Gallium-67 Accumulation Is Characteristic Feature of Autoimmune Pancreatitis. Pancreas, 27, 20. https://doi.org/10.1097/00006676-200307000-00003

[20] Zen, Y. and Nakanuma, Y. (2010) IgG4-Related Disease: A Cross-Sectional Study of 114 Cases. The American Journal of Surgical Pathology, 34, 1812-1819. https://doi.org/10.1097/PAS.0b013e3181f7266b

[21] Soliotis, F., Mavragani, C.P., Plastiras, S.C., et al. (2014) IgG4-Related Disease: A Rheumatologist's Perspective. Clinical and Experimental Rheumatology, 32, 724.

[22] Ikeda, T., Oka, M., Shimizu, H., et al. (2013) IgG4-Related Skin Manifestations in Patients with IgG4-Related Disease. European Journal of Dermatology, 23, 241. https://doi.org/10.1684/ejd.2013.1958

[23] Charrow, A., Imadojemu, S., Stephen, S., et al. (2016) Cutaneous Manifestations of IgG4-Related Disease (RD): A Systematic Review. Journal of the American Academy of Dermatology, 75, 197. https://doi.org/10.1016/j.jaad.2016.01.046

[24] Umemura, T., Zen, Y., Hamano, H., et al. (2007) IgG4 Associated Autoimmune Hepatitis: A Differential Diagnosis for Classical Autoimmune Hepatitis. Gut, 56, 1471. https://doi.org/10.1136/gut.2007.122283 